\title{
Distribution sur les membres interdépendants - une analyse de la coordination nue en français
}

\author{
Makoto Kaneko ${ }^{1}$ \\ Université d'Okayama, 3-1-1 Tsushima-naka, Kita-ku, Okayama, 700-8530, Japon
}

\begin{abstract}
Résumé. Cette étude vise à clarifier les conditions légitimatrices de la coordination nue en français, en examinant sa compatibilité avec différents types de prédicats pluriels. Il est soutenu que cette construction n'est pas toujours légitimée lexicalement par une relation stéréotypique entre les conjoints, et exige que leur interdépendance soit établie par la comitativité, la relation symétrique ou la dépendance épistémique. La dépendance épistémique signale que le locuteur peut prévoir une propriété d'un membre à travers des connaissances acquises sur une propriété de l'autre membre. Cette hypothèse permet de rendre compte de l'incompatibilité de la coordination nue avec deux types de prédicats pluriels : i) prédicat collectif qui n'introduit pas de division des membres (ex. être un couple) ; ii) prédicat distributif dont le sujet dénote des membres totalement indépendants (ex. être respectivement nés). Deux hypothèses sont ensuite proposées sur l'origine de cette condition légitimatrice : i) les déterminants présupposent l'uniformité des membres. Leur absence indique inversement la non-uniformité des membres. C'est pour confirmer cette non-uniformité référentielle que la division des membres est requise ; ii) la coordination des noms nus reçoit par défaut une lecture intersective. Pour éviter à cette lecture intersective, la coordination nue exige qu'une relation de paire soit établie entre les conjoints. C'est pour justifier une telle relation de paire qu'une interdépendance est invoquée entre les conjoints.
\end{abstract}

\begin{abstract}
Distribution over interdependent members - an analysis of French bare coordination. This study aims to clarify licensing conditions of French bare coordination, by examining its compatibility with different types of plural predicates. It is argued that bare coordination is not always licensed by a stereotypical relation between its conjuncts, and requires that their interdependence be established by togetherness, symmetry or epistemic dependency. The epistemic dependency signals that the speaker can predict a property of a member through knowledge about a property of another member. This hypothesis allows us to account for the incompatibility of bare coordination with two types of plural predicates: i) collective predicate which does not introduce any division between the members (ex. être un couple); ii) distributive predicate whose subject
\end{abstract}

\footnotetext{
${ }^{1}$ Auteur de correspondance : kaneko06@cc.okayama-u.ac.jp
} 
refers to totally independent members (ex. être respectivement nés). Two hypotheses are next advanced regarding the origin of this licensing condition: i) determiners presuppose the uniformity of the members. Their lack inversely signals the non-uniformity of members. It is to confirm such referential non-uniformity that the division of the members is required; ii) bare coordination is by default interpreted as set-intersection. To circumvent the intersective reading, argumental bare coordination requires that its conjuncts are interpreted as forming a pair relation. It is to justify such pair relation that some interdependency is invoked between the conjuncts.

\section{Introduction}

Cette étude a pour objectif de mettre au clair les conditions légitimatrices de la coordination nue en français, en examinant les types de prédicats pluriels compatibles avec cette construction. Il est bien connu que les noms nus en français sont en principe exclus dans une position argumentale. Toutefois, la coordination autorise leur occurrence, comme l'indique le contraste d'acceptabilité entre (1a) et (1b).

(1)a. *Linguistes se sont toujours accordés à reconnaître que [...].

b. Philosophes et linguistes ${ }^{2}$ se sont toujours accordés à reconnaître que sans le secours des signes, nous serions incapables de distinguer deux idées d'une façon claire et constante. (Saussure, Cours de linguistique générale, Ch.IV, §1)

Les travaux antérieurs s'accordent pour soutenir que les conjoints y forment «un tout» (Riegel et al., 1999 : 164 ; Gary-Prieur, 2011 : 46) ou « un tout structuré » (Curat, 1999 : 319). Lambrecht $(1984)^{3}$ et Haspelmath $(2007)^{4}$, entre autres, arguent qu' « un tout » est construit lexicalement par une relation stéréotypique et conventionalisée. Cette analyse en termes de la légitimation lexicale pourrait s'appliquer aux conjoints nus, mari et femme en (2). Toutefois, la difficulté de (3b), qui inclut les mêmes conjoints, indique que la relation stéréotypique ne permet pas toujours l'absence d'article.

(2) Mari et femme se respectent toujours comme des invités honorés.

(http://mcdebaecque.org/ressource/enseignement-sur-lamour-thich-nhathanh/\#.VmVMARaheM8)

(3)a. Le mari et la femme sont respectivement nés le 5 juillet 1944 et le 4 juin 1947. (http://france.globe24h.com/lex/judiciaire/007/076/07631/0007631378.shtml)

b. $\quad$ ?Mari et femme sont respectivement nés le 5 juillet 1944 et le 4 juin 1947.

D'autre part, Märzhäuser $(2013$; 2014) soutient qu'un « tout » est formé contextuellement lorsque les conjoints sont mis dans le même cadre énonciatif. Selon cet auteur, le cadre énonciatif est établi i) par «la comitativité », c'est-à-dire, «la participation simultanée » des conjoints et leur «interaction dans l'événement», ou ii) par «la relation symétrique » entre les conjoints. Par exemple, en (4a), les conjoints, homme et voiture, établissent une

\footnotetext{
${ }^{2}$ Dans ce qui suit, les éléments coordonnés seront mis en italique.

3 «In the regular conjoined structure, the second member is simply added to the first in a cumulative fashion; but in the BB [Bare Binominal] the two members are unified into a single semantic complex. » (Lambrecht, $1984: 756)$

4 « The conjuncts habitually go together and can be said to form some conventionalized whole or conceptual unit. » (Haspelmath, $2007: 21$ )
} 
relation comitative par le fait de s'engager dans le même événement ${ }^{5}$, tandis qu'en (4b), une relation «symétrique » par rapport à la vie et à la mort est contextuellement construite entre la maison et le livre. Il est à noter que ces deux exemples vont à l'encontre de la légitimation lexicale : il est difficile de dire que, dans les phrases soulignées en (4a) et (4b), la relation entre l'homme et la voiture et celle entre la maison et le livre sont conventionalisées et stéréotypiques.

(4)a. Il vérifie son revolver, dégaine son coutelas de combat, compte une douzaine de grenades à portée de main, embraie et arrache littéralement sa jeep du sol. Il bondit. Non, il vole. Vingt minutes plus tard, homme et voiture arrivent au camp. (Gigon, Les Américains face au Vietcong, 1965, cité dans Märzhäuser, $2014: 2506)$

b. « 'L'homme construit des maisons parce qu'il est vivant, mais il écrit des livres parce qu'il se sait mortel.' (Pennac, Comme un Roman) (...) Maison et livre invitent pourtant tous deux à interroger selon leur modèle notre habitation du monde. » (http://fabula.org/actualite/article19789.php, cité dans Märzhäuser, $2014: 2507)$

Märzhäuser (2013) fait par ailleurs remarquer que certaines langues disposent d'un coordinateur comitatif, qui est dérivé de la préposition avec. Une telle coordination comitative est illustrée par l'exemple (5a) en russe qui implique un coordinateur comitatif, $s$ ('avec'), suivi d'un syntagme nominal mis au cas instrumental. Selon McNally (1993), (5a) n'admet que la lecture comitative mise en évidence par l'adverbe ensemble et non pas la lecture distributive, à la différence de la coordination ordinaire avec un coordinateur, $i$ ('et'), comme en (5b).
(5)a. Anna $s \quad$ Petej
pridut. (McNally, 1993: 353)
A.-NOMINATIF avec P.-INSTRUMENTAL viennent

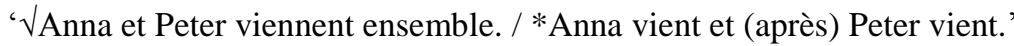
b. Anna i Petja pridut. (ibid.)
A.-NOMINATIF et P.-NOMINATIF viennent

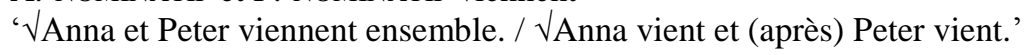

Märzhäuser (2013) suggère que le français, dépourvu d'une telle distinction lexicale, recourt à la coordination nue pour incarner le type comitatif de coordination ${ }^{6}$. Il reste pourtant à clarifier la nature de la comitativité en question, d'autant plus que la comitativité exprimée par ensemble est sous-classifiée en types adverbial et adnominal, respectivement illustrés par (6a) et (6b).

(6)a. Jean et Marie ont acheté une maison ensemble. (traduit de Lasersohn, 1998 : 273)

b. Jean et Marie ensemble ont acheté une maison. (ibid.)

La notion de «symétrie contextuelle » à laquelle Märzhäuser (2013) recourt pour rendre compte de la relation entre la maison et le livre en (4b) nécessitera aussi plus de

\footnotetext{
5 «The two Ns have a special 'togetherness' - they are two distinct elements acting as a unit (in the situation referred to). » (Märzhäuser, $2013: 297$ )

6 "Many languages possess a comitative and, which developped out of the preposition 'with' for noun coordination, and a different coordinator for sentences and verb phrases. [...] comitative and might preserve some of the semantic weight of the preposition 'with'. Languages with only one coordinating and for NPs and VPs need different linguistic strategies to express this semantic aspect of 'togetherness'. The construction of CBN [Coodinated Bare Nouns] sets the coordinates aside from regular noun phrases with determiners: they are an econominal linguistic means to say that two entities appear together and are closely related. » (Märzhäuser, $2013: 297$ )
} 
clarification. Les prédicats réciproques, comme se respecter en (2) (repris en (7a)), les prédicats implicitement comitatifs, comme arriver au camp en (4a) (repris en (8a)) ainsi que la lecture interne ${ }^{7}$ de certains adjectifs, comme contraires en (9a), évoquent une «symétrie » par le fait que leurs arguments se permutent, comme le représentent (7b), (8b) et (9b) : (7b) signifie que, si le mari respecte sa femme, celle-ci le respecte aussi. Il en est de même pour (6b) et (8b) (cf. Cadiot, 1997). En revanche, le prédicat distributif inviter à interroger notre habitation du monde en (4b) (repris en (10)) ne se laisse pas paraphraser avec la permutation des arguments.

(7)a. Mari et femme se respectent toujours comme des invités honorés. (=(2))

b. $\operatorname{RESPECTER}(\mathrm{m}, \mathrm{f}) \rightarrow \operatorname{RESPECTER}(\mathrm{f}, \mathrm{m})$.

(8)a. Homme et voiture arrivent (ensemble) au camp. (=(4a))

b. ARRIVER AU CAMP AVEC $(h, v) \rightarrow$ ARRIVER AU CAMP AVEC $(v, h)$.

(9)a. S'agissant des indicateurs de mesure de la pauvreté, France et Allemagne ont connu des évolutions contraires en ce qui concerne le taux de pauvreté relative qui, depuis 2000, a augmenté au sein de l'Union européenne. (Rapport de la cour des comptes : 38 )

http://www.ladocumentationfrancaise.fr/var/storage/rapportspublics/114000118.pdf

b. CONNAITRE UNE EVOLUTION CONTRAIRE A $(\mathrm{f}, \mathrm{a}) \rightarrow$ CONNAITRE UNE EVOLUTION CONTRAIRE A $(\mathrm{a}, \mathrm{f})$

(10) Maison et livre invitent à interroger selon leur modèle notre habitation du monde. $(=(4 b))$

Dans ce qui suit, je montrerai que l'acceptabilité et l'inacceptabilité des exemples qui impliquent tous un prédicat distributif, comme $(3 b)$ et $(4 b)$, nous amènent à remettre en cause la dichotomie courante entre prédicats collectifs et distributifs, et je proposerai de réinterpréter la notion de «symétrie contextuelle » préconisée par Märzhäuser (2013; 2014) au moyen de la « dépendance épistémique » avancée par Mari (2005a,b), et de rendre compte des conditions légitimatrices de la coordination nue en termes de l'interdépendance entre les conjoints.

La discussion est organisée dans l'ordre suivant. Je passerai d'abord en revue des travaux antérieurs sur la comitativité (section 2) et sur la dichotomie entre les prédicats collectifs et distributifs (section 3), en montrant qu'aucune de ces analyses ne peut toute seule pleinement rendre compte des conditions légitimatrices de la coordination nue. J'avancerai ensuite une hypothèse selon laquelle, tout en dénotant des membres divisés, les conjoints nus forment un «tout» par l'interdépendance qui est établie entre les membres dénotés par la «symétrie logique » (définie par la permutabilité des arguments) ou par la «symétrie contextuelle» (redéfinie par la dépendance épistémique) (section 4). J'examinerai ensuite pourquoi une telle restriction est imposée sur la coordination nue (section 5). La section 6 récapituera les principaux résultats de cette étude.

\section{La comitativité}

Cette section discutera deux analyses précédentes de la comitativité : Moltmann (2004) (section 3.1) et Lasersohn (1998) (section 3.2).

\footnotetext{
${ }^{7}$ Dans la lecture interne, on trouve les arguments d'un adjectif à l'intérieur de la phrase, ce qui n'est pas le cas dans la lecture externe. Celle-ci est illustrée par contraire en (i).

(i) L'Allemagne a baissé l'impôt sur les sociétés. La France a pris une politique fiscale contraire.
} 


\subsection{Comitativité adnominale : Moltmann (2004)}

Moltmann (2004) observe que la comitativité adnominale doit mettre en cuvre une mesure numérique ('numerical measurement'), comme 100,000 dollars en (11a) : l'absence de mesure numérique dégrade l'acceptabilité de cet emploi, comme en (11b). Moltmann (2004 : 295) argue ainsi que « la contribution de together ('ensemble') [de type adnominal] est de montrer que l'ensemble de la somme apportée par chaque membre aboutit à la mesure numérique indiquée ${ }^{8}$. Ainsi, l'emploi adnominal de ensemble en (11a) signale que la somme du salaire de Jean et de celui de Marie est égale à 100. 000 dollars.

(11)a. Jean et Marie ensemble gagnent 100. 000 dollars par mois. (traduit de Moltmann, 2004 : 295)

b. \#Jean et Marie ensemble sont payés par mois. (idem. : 294)

Cette contrainte n'est pas imposée à la coordination nue, comme le montre l'absence de mesure dans les exemples attestés en $(12 \mathrm{a}, \mathrm{b})$. Ceci suggère que la comitativité transmise par la coordination nue n'est pas de type adnominal.

(12)a. Homme et voiture arrivent (ensemble) au camp. (=(4a))

b. Réforme fiscale et élaboration d'une nouvelle reconnaissance de la citoyenneté allaient de pair. (Rosanvallon, La société des égaux : 378)

\subsection{Comitativité adverbiale : Lasersohn (1998)}

Je vérifierai maintenant si la comitativité dont il s'agit dans la coordination nue correspond au type adverbial. C'est Lasersohn (1998) qui distingue quatre lectures de la comitativité adverbiale : i) action collective ${ }^{9}$; ii) action coordonnée ; iii) accompagnement ; iv) proximité spatio-temporelle ${ }^{10}$. Chacune des quatre lectures est illustrée en (13a-d).

(13)a. Jean et Marie ont acheté une maison ensemble. (=(16a)) [action collective]

b. Jean et Marie travaillent ensemble. (idem. : 278) [action coordonnée]

c. Jean et Marie sont sortis ensemble. (ibid.)

[accompagnement]

d. Jean et Marie étaient assis ensemble. (ibid.) [proximité spatio-temporelle]

Les exemples attestés en (14a-d) montrent que la coordination nue peut certes incarner ces quatre lectures.

\footnotetext{
8 « The contribution of together is to specify that adding up the measurement of the members of the group yields the measurement. » (Moltmann, $2003: 295$ ).

${ }^{9}$ La différence entre l'action collective et l'action coordonnée est que la coordination est non booléenne dans le premier cas et booléenne dans le second : (13a) qui exprime une action collective n'implique ni que Jean a acheté une maison, ni que Marie a acheté une maison, comme en (ia) ; (13b), qui transmet une action coordonnée, implique d'autre part que Jean travaille et que Marie travaille, comme en (ib).

(i)a. Jean et Marie ont acheté une maison ensemble. $\nrightarrow$ Jean a acheté une maison et Marie a acheté une maison.

b. Jean et Marie travaillent ensemble. $\rightarrow$ Jean travaille et Marie travaille.

${ }^{10}$ Lasersohn (1998) analyse, en fait, l'exemple en (13d) incluant « être assis ensemble » comme incarnant la proximité spatiale (paraphrasée par côte à côte) et en distingue l'exemple en (i) qui illustre, selon cet auteur, la simultanéité temporelle (paraphrasée par en même temps). Mais comme le note Moltmann (2003 : 309), ces deux emplois sont très souvent observés en même temps, et sont difficiles à distinguer. Ils ne sont donc pas distingués dans cette étude.
}

(i) Jean et Marie se sont levés ensemble. (traduit de Lasersohn, 1998 : 278) 
(14)a. À Toulouse, étudiants et professeurs inventent le 'campus de futur'. (http://etudiant.lefigaro.fr/les-news/actu/detail/article/a-toulouse-etudiants-etprofesseurs-inventent-le-campus-du-futur-9508/) [action collective]

b. Dans les soutes, ils se démenaient et ils grognaient sérieusement. Ils étaient à la limite de leurs forces. Soutiers et chauffeurs [...] chargeaient le charbon dans les chariots et le transportaient à bâbord. (Peisson, Parti de Liverpool, 1932 : 208) [action coordonnée]

c. Réforme fiscale et élaboration d'une nouvelle reconnaissance de la citoyenneté allaient de pair. (=(12b)) [accompagnement]

d. Il a posé verres et bouteille sur le sol. (Benoziglio, Cabinet portrait, 1980, cité par Mulder, 2009 : 62) [proximité spatio-temporelle]

On atteste toutefois des exemples comme en $(15 \mathrm{a}, \mathrm{b}, \mathrm{c})$ où on ne reconnaît ni la symétrie logique (définie par la permutabilité des arguments), ni la comitativité adverbiale.

(15)a. Ainsi, au cours des vingt dernières années, France et Allemagne ont diminué (\#ensemble) leur taux marginal supérieur d'impôt sur le revenu. (Rapport de la cour des comptes : 85)

http://www.ladocumentationfrancaise.fr/var/storage/rapportspublics/114000118.pdf

b. Hommes et femmes portaient (\#ensemble) aux lèvres des labrets. (Lévi-Strauss, Triste tropiques, 1955, cité par Curat, 1999 : 318)

c. Certes, le savant a raison de ne voir dans ses appareils que les théories qu'ils matérialisent, dans les produits employés, que les réactions qu'ils permettent, et de postuler la validité universelle de ces théories et de ces réactions, mais pour le vivant, appareils et produits sont (\#ensemble) des objets parmi lesquels il se meut comme dans un monde insolide. (Canguilhem, cité par Benetti, $2008: 102)$

Ces exemples ne sont d'ailleurs pas exceptionnels : dans mon corpus consistant en 135 exemples attestés de coordination nue en position sujet ou objet, 89 cas (soit 66\%) incarnent un des quatre emplois de la comitativité ; 30 cas (22\%) mettent en cuvre d'autres types de symétrie logique (discernée par la permutabilité des arguments), tels que la réciprocité, la lecture interne de contraires, différents, mêmes, pareils, etc. ; 16 cas (12\%) contiennent un prédicat distributif qui ne semble évoquer ni la symétrie logique, ni la comitativité, comme en (4b) et (15a-c).

Tableau 1

\begin{tabular}{|c|c|c|}
\hline comitativité $:(4 \mathrm{a}),(14 \mathrm{a}-\mathrm{d})$ & symétrie logique $:(1 \mathrm{~b}),(2),(9 \mathrm{a})$ & autres cas $:(4 \mathrm{~b}),(15 \mathrm{a}-\mathrm{c})$ \\
\hline 89 cas $(66 \%)$ & 30 cas $(22 \%)$ & 16 cas $(12 \%)$ \\
\hline
\end{tabular}

Pour mieux mettre en place les troisièmes cas, je réviserai, dans la prochaine section, deux études qui portent sur la dichotomie entre les prédicats collectifs et distributifs. 


\section{La dichotomie entre prédicats collectifs et distributifs}

Cette section envisagera deux analyses qui revisitent la dichotomie entre les prédicats collectifs et distributifs : Brisson (2003) (section 3.1) et Mari (2005a,b ; 2012) (section 3.2).

\subsection{Sous-classification des prédicats collectifs : Brisson (2003)}

Brisson (2003) observe que les prédicats collectifs ne se comportent pas de manière uniforme en ce qui concerne la compatibilité avec tous / toutes, et que cette (in)compatibilité est solidaire de l'Aktionsart des prédicats : en principe, les prédicats qui incarnent une activité ou un accomplissement sont compatibles avec tous / toutes, ce qui n'est pas le cas pour les prédicats qui expriment un état ou un achèvement, comme en (16ad).

(16)a. *Tous les garçons sont un grand groupe. (traduit de Brisson, 2003 : 146) [état]

b. Tous les garçons ont emporté le piano pendant une heure. (ibid.) [activité]

c. *Tous les étudiants ont élu un président. (idem. : 147) [achèvement]

d. Tous les garçons forment un grand groupe. (ibid.) [accomplissement]

Brisson (2003) fait aussi remarquer qu'un état et un achèvement deviennent compatibles avec tous / toutes lorsqu'ils transmettent une symétrie logique (c'est-à-dire, lorsqu'ils permettent la permutation des arguments), y compris la réciprocité et la comitativité, comme en $(17 \mathrm{a}, \mathrm{b}, \mathrm{c})$.

(17)a. Tous les chiens sont pareils (ibid.)

[état symétrique]

b. Tous les garçons se sont reconnus l'un l'autre (ibid.) [achèvement réciproque]

c. Tous les vols sont arrivés ensemble. (ibid.) [achèvement comitatif]

Pour expliquer cette différence entre les prédicats collectifs, Brisson (2003) soutient que l'occurrence de tous / toutes présuppose une division des membres : les prédicats collectifs d'état (ex. être un grand groupe) et d'achèvement (ex. élire un président) exigent, pour leur sujet, une entité plurielle indivisible, tandis que ceux d'activité (ex. emporter le piano) et d'accomplissement (ex. former un grand groupe) demandent une division préalable des membres, ce qui est aussi le cas pour les prédicats logiquement symétriques.

Examinons maintenant si la sous-classification des prédicats collectifs avancée par Brisson (2003) peut décrire les conditions légitimatrices de la coordination nue. En (18) qui met en cuvre un prédicat d'état, être un groupe, les conjoints nus, France et Allemagne, sont moins acceptables que les conjoints déterminés, la France et l'allemagne, tandis qu'en $(19 \mathrm{a}, \mathrm{b})$ dont le prédicat est logiquement symétrique, les mêmes conjoints nus sont acceptés. Ceci prouve que la coordination nue exige certes une division des membres.

(18) Il ne faut pas se dire qu'il n'y a pas de problèmes quand il y en a. Mais encore une fois, l'idée c'est de dire : voilà, \{la France et l'Allemagne sont un couple, la France et l'Allemagne sont le moteur de l'Europe / ?? France et l'Allemagne sont un couple, France et Allemagne sont le moteur de l'Europe $\}$; il n'y a pas d'alliance de revers possible, il n'y a pas d'alternative ; si nous fonctionnons chacun de notre côté, alors c'est l'Europe tout entière qui va en pâtir. (http://discours.vie-publique.fr/notices/013000660.html) [prédicat d'état]

(19)a. S'agissant des indicateurs de mesure de la pauvreté, France et Allemagne ont connu des évolutions contraires en ce qui concerne le taux de pauvreté relative $(=(9 \mathrm{a}))$ [prédicat symétrique d'accomplissement]

b. France et Allemagne continuent de coopérer pour approfondir la construction européenne. 
(http://fr.wikipedia.org/wiki/Relations_entre_1\%27Allemagne et la France\#Ge rhard_Schr.C 3.B6der_et Jacques_Chirac_.281998.E2.80.932005.29) [prédicat comitatif d'activité]

D'autre part, le contraste d'acceptabilité entre (3b) (repris en (20a)) et (15a) (repris en (20b)), qui impliquent tous les deux un prédicat distributif, indique que la présence ou l'absence de division des membres n'est toute seule pas suffisante pour décrire pleinement les comportements de la coordination nue.

(20)a. ?Mari et femme sont respectivement nés le 5 juillet 1944 et le 4 juin 1947. $(=(3 b))$ [prédicat distributif]

b. Ainsi, au cours des vingt dernières années, France et Allemagne ont diminué (\#ensemble) leur taux marginal supérieur d'impôt sur le revenu. $(=(15 \mathrm{a}))$ [prédicat distributif]

Pour rendre compte de la différence entre ces deux exemples, la section suivante présentera une autre tentative de remise en cause de la dichotomie des prédicats collectifs et distributifs.

\subsection{Distinction tripartite : Mari (2005a,b ; 2012)}

Mari (2012) propose, développant ses propres analyses dans Mari (2005a,b) et suivant l'analyse de Hackl (2002), de distinguer trois types de prédicats pluriels : i) véritables prédicats collectifs ('genuine collective predicates'), comme être un couple, être nombreux ; ii) prédicats individuels pluralisés ('pluralized individual predicates'), tels que être étudiants, avoir les yeux bleus ; iii) prédicats inhéremment pluriels ('inherently plural predicates'), comme être des voisins, se rencontrer. Le premier type exige, pour son sujet, une pluralité indivisible ; le second type présuppose, au contraire, la division des référents du sujet et s'applique à chacun d'entre eux sans relation interne entre eux; le troisième type présuppose aussi la division des membres et porte sur eux avec leur interdépendance. Par exemple, i) le fait que «Jean et Marie sont un couple. »n'implique pas que «Jean est un couple. »; ii) le fait que «Jean et Marie ont les yeux bleus. » implique que «Jean a les yeux bleus. » indépendamment du fait que «Marie a les yeux bleus. »; iii) le fait que «Jean et Marie sont des voisins. » implique que «Jean est un voisin de Marie. » et inversement.

Les véritables prédicats collectifs chez Mari (2012) correspondent ainsi aux prédicats collectifs d'état (ex. être un grand groupe) et d'achèvement (ex. élire un président) chez Brisson (2003), tandis que les prédicats inhéremment pluriels chez Mari (2012) ne coïncident pas exactement avec les prédicats collectifs d'activité (ex. emporter le piano) et d'accomplissement (ex. former un grand groupe) chez Brisson (2003). Cruciellement, selon Mari (2012), la mise en relation des membres par les prédicats inhéremment pluriels peut être de nature épistémique et fondée sur les attentes du locuteur concernant l'état des choses. Par exemple, le prédicat, marcher le long de la plage en (21a), est ambigu entre les lectures individuelle pluralisée et inhéremment plurielle. Selon la première lecture, le locuteur ne transmet qu'une simple observation des comportements des deux personnes ; suivant la seconde lecture, le comportement de Jean apporte au locuteur une information utile pour anticiper celui de Marie. (21a) signale dans ce cas que le locuteur assume que les deux personnes marcheraient ensemble le long de la même trajectoire si l'état des choses continue tel qu'il est dans le monde actuel.

(21)a. Jean et Marie marchent le long de la plage. (traduit de Mari, 2005b : 190)

b. $\left[j \otimes_{\text {MARCher (ENSEMble) }} m\right]^{\mathrm{w}, \mathrm{t}}$ si et seulement si $\forall \mathrm{w}^{\prime} \in \operatorname{In} r(\mathrm{w}, \mathrm{t})$ [SUIVRE LA DIRECTION DE $\mathrm{j}\left(\mathrm{j}, \mathrm{w}^{\prime}\right) \rightarrow$ SUIVRE LA DIRECTION DE $\mathrm{j}\left(\mathrm{m}, \mathrm{w}^{\prime}\right)$ ] (inspiré par Mari, 


\section{$2012: 156)$}

Mari (2005b) baptise une telle mise en relation dépendance épistémique. En simplifiant la formalisation de Mari (2012), la dépendance épistémique en (21a) est représentée par (21b) : (21b) signale que Jean et Marie sont épistémiquement dépendants (noté par le symbole $\otimes)$ par rapport au prédicat, marcher le long de la plage, si et seulement si, lorsque l'état des choses continue tel qu'il est dans le monde actuel, c'est-à-dire, dans tous les mondes inertes (noté par Inr) accessibles du monde actuel $w$, si Jean suit une direction, Marie suit la même direction.

Il est à noter que Mari (2005a,b) suggère aussi de réinterpréter la comitativité en termes de la dépendance épistémique. Elle observe ainsi que l'adverbe comitatif ensemble n'est pas toujours accepté lorsque le prédicat verbal transmet une proximité spatio-temporelle, comme l'illustrent le contraste d'acceptabilité entre (22a) et (22b) et celui entre (23a) et (23b). Selon Mari (2005a), en (22a), la localisation des verres apporte au locuteur une information utile pour connaître celle des carafes, tandis qu'en (22b), la localisation de la porte ne lui sert pas à spécifier celle de la fenêtre ; en (23a), le locuteur peut prévoir que, si Jean reste dans la salle d'attente, Marie aussi, et que, si Jean se déplace, Marie le suivra. D'autre part, en (23b), la localisation de Jean ne sert pas du tout au locuteur pour prévoir celle de Chirac.

(22)a. Les verres et la carafe sont ensemble dans le buffet. (Mari, 2005a : 88)

b. ??La porte et la fenêtre sont ensemble dans le salon. (ibid.)

(23)a. Jean et Marie sont ensemble dans la salle d'attente. (Jayez \& Mari, 2005 : 157)

b. ??Jean et Chirac sont à Paris ensemble. (Mari, 2005a : 88)

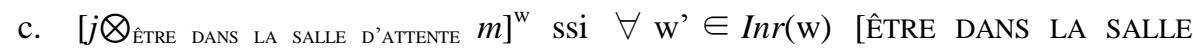
D’ATTENTE $\left(j, w^{\prime}\right) \rightarrow$ ÊTRE DANS LA SALLE D’ATTENTE $\left.\left(m, w^{\prime}\right)\right]$

La sémantique de (23a) est représentée par (23c) : (23c) énonce que Jean et Marie sont épistémiquement dépendants par rapport au prédicat localisant être dans la salle d'attente si et seulement si, dans tous les mondes inertes $w^{\prime}$ accessibles du monde actuel $w$, si Jean est dans la salle d'attente, Marie y est aussi. Je profiterai, dans la section suivante, de la notion de la dépendance épistémique pour rendre compte des exemples problématiques de coordination nue comme $(4 \mathrm{~b})$ et $(15 \mathrm{a}, \mathrm{b}, \mathrm{c})$.

\section{Hypothèse : distribution sur les membres interdépendants}

J'avancerai maintenant, en me référant aux travaux antérieurs présentés dans les sections précédentes, les hypothèses $(24 \mathrm{a}, \mathrm{b})$ pour rendre compte des comportements de la coordination nue, notés ci-dessus.

(24)a. La coordination nue dans une position argumentale présuppose une division des membres.

b. Elle exige que le prédicat verbal établisse une interdépendance entre les membres divisés.

\section{1 (In)compatibilité avec différents prédicats pluriels}

Ces hypothèses permettent d'expliquer la compatibilité et l'incompatibilité de la coordination nue avec différents prédicats pluriels : i) la coordination nue n'est pas compatible avec les véritables prédicats collectifs (Mari, 2005) ou prédicats collectifs d'état (Brisson, 2003), comme être un couple en (25), car ceux-ci exigent, pour leur sujet, une pluralité indivisible et ne permettent donc pas la division des membres, contrairement à (24a). 
(25) ??France et Allemagne sont un couple. (=(18)) [prédicat collectif véritable d'état]

ii) Elle est compatible avec les prédicats inhéremment pluriels de type réciproque, comme se respecter en (26a), ou de type comitatif, comme coopérer en (27a), car ces prédicats présupposent, conformément à $(24 a, b)$, une division des membres et établissent leur interdépendance par leur permutabilité, comme le montrent les représentations sémantiques en (26b) (qui veut dire que si le mari respecte sa femme, celle-ci le respecte aussi) et (27b) (qui signifie que si la France coopère avec l'Allemagne, celle-ci coopère avec elle). Il en est de même pour les prédicats mettant en cuvre la lecture interne de certains adjectifs, comme contraires, différents, mêmes, pareils, etc.

(26)a. Mari et femme se respectent toujours comme des invités honorés. (=(2)) [prédicat inhéremment pluriel de type réciproque]

b. RESPECTER $(\mathrm{m}, \mathrm{f}) \rightarrow \operatorname{RESPECTER}(\mathrm{f}, \mathrm{m})$

(27)a. France et Allemagne continuent de coopérer pour approfondir la construction européenne. $(=(19 b))$ [prédicat inhéremment pluriel de type comitatif]

b. COOPERER AVEC $(f, a) \rightarrow \operatorname{COOPERER} \operatorname{AVEC}(a, f)$

iii) Elle est compatible avec certains prédicats distributifs, comme devenir plus grands en (28a), diminuer le taux marginal supérieur d'impôt sur le revenu en (29a) et inviter à interroger notre habitation du monde en (30a), car ces prédicats satisfont la condition d'interdépendance en (24b) en introduisant une dépendance épistémique : en (28a), le locuteur peut prévoir que, si les loups deviennent plus grands à mesure qu'on avance vers le nord, il en est de même pour les ours, ce qui est représenté en (28b) et glosé en (28c).

(28)a. Loups et ours deviennent plus grands à mesure qu'on avance vers le nord. (Roodenburg, 2005: 105) [prédicat distributif avec une dépendance épistémique]

b. $\quad\left[l \bigotimes_{\text {DeVenir plus grands }} o\right]^{\mathrm{w}}$ ssi $\forall \mathrm{w}^{\prime} \in \operatorname{In} r(\mathrm{w})$ [DEVENIR PlUS GRANDS $\left(1, \mathrm{w}^{\prime}\right) \rightarrow$ DEVENIR PLUS GRANDS $\left.\left(\mathrm{o}, \mathrm{w}^{\prime}\right)\right]$

c. En mots : les loups et les ours sont épistémiquement dépendants par rapport au prédicat distributif, devenir plus grands, si et seulement si, dans tous les mondes inertes $w^{\prime}$, accessibles du monde actuel $w$, si les loups deviennent plus grands à mesure qu'on avance vers le nord, il en est de même pour les ours.

De même, en (29a), les connaissances sur la politique fiscale de la France permettent au locuteur de prévoir celle de l'Allemagne, comme l'indique (29b) et le glose (29c).

(29)a. Au cours des vingt dernières années, France et Allemagne ont diminué leur taux marginal supérieur d'impôt sur le revenu. (=(15a)) [prédicat distributif avec une dépendance épistémique]

b. $\left[f \bigotimes_{\text {Diminuer le tauX Marginal supérieur d'Impôt sur le ReVenu }} a\right]^{\mathrm{w}}$ ssi $\forall \mathrm{w}^{\prime} \in \operatorname{In} r(\mathrm{w})$ [DIMINUER LE TAUX MARGINAL SUPÉRIEUR D'IMPÔT SUR LE REVENU (f, w') $\rightarrow$ DIMINUER LE TAUX MARGINAL SUPÉRIEUR D'IMPÔT SUR LE REVENU (a, w')]

c. En d'autres termes : la France et l'Allemagne sont épistémiquement dépendants par rapport au prédicat distributif diminuer le taux marginal supérieur d'impôt sur le revenu si et seulement si, dans tous les mondes inertes $w^{\prime}$, accessibles du monde actuel $w$, si la France diminue le taux marginal supérieur d'impôt sur le revenu, l'Allemagne fera la même chose.

Enfin, en (30a) pour lequel Märzhäuser (2014) préconise la «symétrie contextuelle », dans un contexte construit discursivement, si la maison nous invite à interroger selon son modèle 
notre habitation du monde, il en est de même pour le livre, ce qui est formalisé en (30b) et glosé en (30c).

(30)a. Maison et livre invitent pourtant tous deux à interroger selon leur modèle notre habitation du monde. $(=(4 b))$ [prédicat distributif avec une dépendance épistémique]

b. $\quad\left[m \bigotimes_{\text {INVITER À InTERroger notre habitation du monde }} l\right]^{\mathrm{w}}$ ssi $\forall \mathrm{w}^{\prime} \in \operatorname{In} r(\mathrm{w})$ [INVITER À INTERROGER NOTRE HABITATION DU MONDE $(\mathrm{m}, \mathrm{w}$ ') $\rightarrow$ INVITER À INTERROGER NOTRE HABITATION DU MONDE (l, w')]

c. En d'autres termes : la maison et le livre sont épistémiquement dépendants par rapport au prédicat distributif inviter à interroger notre habitation du monde si et seulement si, dans tous les mondes inertes $w^{\prime}$, accessibles du monde actuel $w$, si la maison invite à interroger notre habitation du monde, il en est de même pour le livre.

iv) Elle est difficilement compatible avec d'autres prédicats distributifs, comme être respectivement nés en (31), car, tout en présupposant une division des membres, ceux-ci sont totalement indépendants, à l'encontre de (24b) : on ne peut pas prédire, du fait que le mari est né le 5 juillet 1944, que sa femme est née le 4 juin 1947.

(31) ?Mari et femme sont respectivement nés le 5 juillet 1944 et le 4 juin 1947. $(=(3 b))$ [prédicat distributif sans dépendance épistémique]

\subsection{D'autres arguments}

Deux arguments viennent à l'appui des hypothèses en $(24 a, b)$. D'abord, celles-ci rendent compte des différences interprétatives notées par Curat (1999) entre la coordination nue et la coordination déterminée. D'après cet auteur, i) la coordination nue, comme députés et sénateurs, forme « une COMMUNAUTÉ » qui implique « une COMPLÉMENTARITÉ [et] une OPPOSITION » (Curat, 1999 : 316-317) ; ii) la coordination à un seul déterminant, comme les députés et sénateurs, «forme un bloc homogène et cohérent » et «amalgame les référents et écrase les traits de COMPLÉMENTARITÉ et d'OPPOSITION» (ibid.); iii) la coordination à déterminant répété, comme les députés et les sénateurs, « ne constitue pas un ensemble. [...] Il n'y a pas de cohérence nécessaire » (idem. : 64) et «on perd le trait de COMMUNAUTÉ » (idem.: 319). La troisième remarque s'accorde avec le fait que chaque syntagme déterminant (DP en termes génératifs) peut construire sa référence indépendamment.

La différence entre les deux coordinations déterminées est illustrée, selon Curat (1999), par les lectures possibles de (32) et (33) : la coordination à un seul article en (32) permet la lecture en (32a) qui « écrase » l'opposition des deux chambres, mais pas la lecture en (32b) indiquant leur opposition; la coordination à deux articles en (33) accepte les deux lectures.

(32) Les députés et sénateurs ne sont pas d'accord là-dessus. (Curat, 1999 : 64)

a. Les deux chambres sont en désaccord interne là-dessus.

b. *Les deux chambres s'opposent là-dessus.

(33) Les députés et les sénateurs ne sont pas d'accord là-dessus. (ibid.)

a. Les deux chambres sont en désaccord interne là-dessus.

b. Les deux chambres s'opposent là-dessus.

Les hypothèses en $(24 a, b)$ qui préconisent la division des membres et leur interdépendance prédisent que la coordination nue en (34) accepte, inversement à la coordination à un seul article en (32), la lecture qui oppose les deux chambres en (34b), mais pas celle qui «amalgame les référents» en (34a). Cette prédiction est confirmée par mes informateurs du français. 
(34) Députés et sénateurs ne sont pas d'accord là-dessus. (=(47))

a. ??Les deux chambres sont en désaccord interne là-dessus.

b. Les deux chambres s'opposent là-dessus.

En effet, dans les exemples attestés de mon corpus qui incluent la coordination nue, députés et sénateurs, tels que les suivants, les deux chambres sont interprétées comme interdépendants.

(35)a. Retraites : députés et sénateurs pas d'accord sur la hausse de cotisations http://www.toutsurlaretraite.com/retraites-deputes-et-senateurs-pas-d-accordsur-la-hausse-de-cotisations.html

b. L'aile du Midi du château de Versailles devrait, d'ici l'été, accueillir les parlementaires pour une nouvelle révision constitutionnelle. C'est en effet dans le palais de Louis XIV que députés et sénateurs se réunissent pour modifier la loi suprême.

http://www.lefigaro.fr/politique/2013/01/31/01002-20130131ARTFIG00744les-autres-volets-du-chantierconstitutionnel

L'idée de la dépendance épistémique sert aussi à contourner un cas problématique de la coordination comitative (cf. l'introduction). Le polonais dispose, de même que le russe, d'une coordination comitative mettant en œuvre le coordinateur $z$ ('avec'). Or, McNally (1993) observe qu'elle est compatible, comme en (36), avec le prédicat distributif avoir les yeux bruns, qui pourrait être classé parmi les prédicats individuels pluralisés (cf. la section 3.2). Selon McNally, (36) est a priori anormal, mais devient plus acceptable lorsque le prédicat sert de critère pour former un ensemble d'individus qui satisfont la même propriété, avoir les yeux bruns.
Anna
z Piotrem
mają brązowe oczy.
[polonais]

A.-NOMINATIF avec P.-INSTRUMENTAL ont bruns yeux

'Anna et Peter ont les yeux bruns.' (McNally, 1993 : 369)

Je soutiendrai que la coordination comitative est ici invoquée pour indiquer une dépendance épistémique entre les deux personnes : le fait qu'Anna a les yeux bruns permet de prédire que Peter a la même propriété, par exemple en raison de leur parenté.

Toutefois, un de mes informateurs fait remarquer que les conjoints nus, frère et sœur en (37a), ne sont pas pleinement acceptables même lorsqu'ils dénotent des jumeaux et que l'anniversaire de l'un permet donc au locuteur de prévoir celui de l'autre, tandis que les conjoints nus mari et femme en (37b) sont acceptés avec le même prédicat verbal. Cet informateur suggère d'attribuer ce contraste d'acceptabilité au fait que le lien stéréotypique est plus facilement établi entre un mari et sa femme qu'entre un frère et sa sœur.

(37)a. [au cas où il s'agit de deux jumeaux]

?Frère et sœur sont nés le 3 septembre 2003.

b. Mari et femme sont nés le 3 septembre 2003.

Je me référerai, pour expliquer ce contraste, à une remarque de Mari (2005b). Selon elle, l'exemple en (38), incluant l'adverbe comitatif ensemble, met en œuvre une dépendance épistémique entre Jean et Franck même lorsque leurs arrivées au sommet coïncident par hasard et donc que ni leur coordination ni leur accompagnement ne sont évoqués, car la coïncidence faiblement probable de deux états de choses (en occurrence, l'arrivée simultanée au sommet de deux personnes) est épistémiquement frappante et informative ${ }^{11}$.

\footnotetext{
11 «The coincidence of two very short events of the same type is epistemically striking. [...] Togetherness corresponds to an epistemic grouping, that associates events with markedly weak probability of co-occurrence. » (Jayez \& Mari, $2005: 159)$
} 
(38) Jean et Frank ont atteint le sommet ensemble. (Mari, 2005b : 90)

Dans cette optique, (37b) est plus naturelle que (37a), car, tandis que la coïncidence des anniversaires d'un mari et de sa femme est peu probable et fortement informative, celle de deux jumeaux est une chose évidente et n'apporte donc pas assez de valeur informative.

\section{Pourquoi la coordination nue exige-t-elle une distribution sur les membres interdépendants?}

Cette section examinera pourquoi la coordination nue est assujettie aux deux contraintes énoncées en (24a,b), à savoir, i) pourquoi elle exige une division des membres (section 5.1) et ii) pourquoi les membres divisés doivent être interdépendants (section 5.2).

\subsection{Pourquoi la coordination nue exige-t-elle une division des membres ?}

Pour envisager la première question, j'avancerai les deux hypothèses en $(39 a, b)$.

(39) Dans la coordination nominale dans une position argumentale,

a. un seul déterminant exige l'uniformité des membres coordonnés.

b. l'absence de déterminants indique, inversement, la non-uniformité des membres coordonnés.

L'hypothèse en (39a) vient du fait que les déterminants sont des quantifieurs : pour que la quantification par un déterminant soit effectuée, les membres dénotés par un ou des nom(s) doivent être uniformes. Et si l'hypothèse en (39b) est correcte, la division des membres est logiquement impliquée par leur non-uniformité.

Les hypothèses en $(39 a, b)$ s'accordent d'abord avec les remarques des trois auteurs suivants : i) selon Curat (1999) (cf. la section 4.2), la coordination nue met en ceuvre « une COMPLÉMENTARITÉ [et] une OPPOSITION », tandis que la coordination à un seul déterminant «forme un bloc homogène et cohérent »; ii) Roodenburg (2005 : 98) soutient qu' « il est difficile en français de coordonner des $\mathrm{N}$ en mettant en facteur le déterminant, sauf [...] si ces $\mathrm{N}$ forment un groupe naturel »; iii) Benetti (2003: 93) fait remarquer qu'un seul déterminant ne précède une coordination que lorsque les conjoints représentent «un hyperonyme construit extensionnellement par conjonction de deux ou plusieurs hyponymes », comme pins et sapins en (40).

(40) Seul, les pins et sapins avaient conservé leur ramure qui renouvelle de saison en saison sans se dépouiller jamais (Verne, cité dans Benetti, 2008 : 91)

Les hypothèses en $(39 a, b)$ sont par ailleurs appuyées par l'argument empirique suivant. Lorsque les conjoints ne forment pas d'hyperonyme, et ne sont donc pas uniformes, comme dans locataires et propriétaires en (41a), un seul déterminant n'est pas accepté. D'autre part, la coordination nue en (41b) est admis, étant donné que les conjoints établissent, grâce au prédicat réciproque se trouver, une interdépendance.

(41)a. ??Les locataires et propriétaires se retrouvent dans la cour tous les dimanches. (Roodenburg, 2005 : 98)

b. Locataires et propriétaires se retrouvent dans la cour tous les dimanches.

Roodenburg (2005) fait remarquer que la présence d'une relative restrictive a pour effet d'inverser l'acceptabilité de la coordination déterminée, comme en (42a), et celle de la coordination nue, comme en (42b).

(42)a. Les locataires et propriétaires qui le souhaitent se retrouvent dans la cour tous les dimanches. (ibid.) 
b. ??Locataires et propriétaires qui le souhaitent se retrouvent dans la cour tous les dimanches.

Cette inversion d'acceptabilité est expliquée par une remarque de Milner (1979: 91) selon laquelle, dans les cas comme (42a), « les deux N' conjoints désignent un groupe 'mixte' dont l'unité est indiquée dans la relative ». Autrement dit, d'après cet auteur, une relative restrictive sert à unifomiser les membres. L'effet uniformisant de la relative restrictive est aussi confirmé par les exemples incluant un quantifieur numéral ${ }^{12}$. Un seul numéral ne peut pas précéder la coordination nominale dont les référents ne sont pas uniformes, comme professeurs de lycée et étudiants de première année en (43a). L'acceptabilité augmente quand la coordination est accompagnée d'une relative restrictive, comme en (43b).

(43)a. *On a condamné pour menées subversives cinq professeurs de lycée et étudiants de première année. (Milner, 1979 : 91)

b. On a condamné pour menées subversives cinq professeurs de lycée et étudiants de première année qui ont signé la pétition. (ibid.)

Dans cette optique, la faible acceptabilité de (42b) indique que la coordination nue est incompatible avec l'effet uniformisant de la relative restrictive, ce qui confirme la validité de l'hypothèse en (39b) selon laquelle cette construction dénote des membres non uniformes.

\subsection{Pouquoi les conjoints nus doivent-ils être interdépendants ?}

La seconde question est de savoir pourquoi les conjoints nus doivent être interprétés comme interdépendants. Pour aborder cette question, je recours à Le Bruyn \& de Swart (2014). Ces auteurs mettent d'abord en cause l'analyse de Heycock \& Zamparelli (2003) selon laquelle l'occurrence de la coordination nue dans une position argumentale est légitimée par un déterminant implicite (par une projection de DP implicite, en termes génératifs). Pour justifier l'absence de déterminant dans la coordination nue, Le Bruyn \& de Swart (2014) commencent par distinguer la coordination intersective, comme philosophes et linguistes en (44a), de la coordination non intersective dont les conjoints dénotent des individus différents, comme en (44b).

(44)a. Saussure et Benveniste sont à la fois philosophes et linguistes.

b. Philosophes et linguistes se sont toujours accordés à reconnaître que sans le secours des signes, nous serions incapables de distinguer deux idées d'une façon claire et constante. $(=(1 \mathrm{~b}))$

Suivant le cadre de la théorie de l'optimalité selon laquelle une contrainte grammaticale peut être violée lorsqu'elle est en concurrence avec une autre contrainte, ils soutiennent ensuite que, bien que le français soit normalement sujet aux contraintes qui exigent que le nombre grammatical, la référentialité et la définitude soient explicitement marqués par un déterminant, ces contraintes sont mises à l'arrière-plan, dans la coordination nominale, par

\footnotetext{
${ }^{12}$ L'exigence de l'uniformité avec un quantifieur numéral est aussi observée en japonais, langue sans articles. Le japonais dispose d'une marque du pluriel, tati, qui exprime soit une pluralité uniforme, soit une pluralité non uniforme. Lorsque cette marque est combinée avec un nom propre, le syntagme nominal entier exprime par défaut une pluralité non uniforme : Marie-tati ne désigne normalement pas un groupe de personnes qui s'appellent toutes Marie (pluralité uniforme), mais Marie et les autres personnes qui lui sont pragmatiquement associées (pluralité non uniforme). Or, lorsqu'un numéral quantifie Marie-tati à l'intérieur du syntagme nominal, comme en (i), la lecture uniforme est imposée.
(i) san-nin-no Marie-tati
trois-CLASSIFICATEUR-GÉNITIF Marie-PLURIEL
'trois filles qui s'appellent toutes Marie'


une autre contrainte qui commande de ne pas marquer de catégorie fonctionnelle au-dessus de la coordination non intersective.

Pour expliquer comment les conjoints nus, qui devraient dénoter une intersection de deux prédicats, arrivent, dans une position argumentale, à dénoter différents individus, Le Bruyn \& de Swart (2014) recourent à une opération sémantique qu'ils appellent MATCHING FUNCTION, qui prend une intersection de deux prédicats et produit un ensemble de paires. Ceci est illustré, pour philosophes et linguistes en (44b), par (45).

\section{(45) MATCHING FUNCTION : (PHILOSOPHES $\cap$ LINGUISTES) $\Rightarrow$ $($ PHILOSOPHES $\times$ E $\cap$ E $\times$ LINGUISTES $)=($ PHILOSOPHES $\times$ LINGUISTES $)$ ( $E$ représente le domaine contexuellement fourni.)}

La MATCHING FUNCTION s'applique selon les trois étapes suivantes : i) elle établit d'abord un ensemble de paires d'individus dans un domaine $E$, dont le premier et le second membres appartiennent, pour (44), respectivement aux philosophes et à $E$ (noté par PHILOSOPHES $\times E$ ) ; ii) elle établit ensuite un autre ensemble de paires d'individus dans le domaine $E$, dont le premier et le second membres appartiennent respectivement à $E$ et aux linguistes (noté par ExLINGUISTES) ; iii) elle effectue enfin l'intersection des deux ensembles de paires, ce qui donne un ensemble de paires dont le premier et le second membres appartiennent respectivement aux philosophes et aux linguistes (noté par PHILOSOPHES $\times$ LINGUISTES). Le domaine $E$ sert ainsi à former une relation de paire.

Je soutiendrai que c'est l'interdépendance invoquée par le prédicat qui fournit le domaine $E$. Par exemple, pour (44b), le domaine $E$ est fourni par le prédicat symétrique $s$ 'accorder, et correspond à l'ensemble de ceux qui se sont accordés à reconnaître que sans le secours des signes, nous serions incapables de distinguer deux idées d'une façon claire et constante. Supposons que le domaine $E$ consiste, dans un contexte pertinent, en \{philosophes, linguistes, psychologues\}. Selon la MATCHING FUNCTION, on obtient d'abord un ensemble de paires comme en (46a); on obtient ensuite un autre ensemble de paires comme en (46b) ; l'intersection des deux ensembles de paires donne un ensemble de deux paires, ce qui se réduit en l'occurrence à la paire <philosophes, linguistes>, comme en (46c).

(46)a. [[philosophes $\times E]]=\{\langle$ philosophes, linguistes $\rangle,\langle$ philosophes, psychologues $\rangle\}$

b. $[[$ Exlinguistes $]]=\{\langle$ philosophes, linguistes $\rangle,\langle$ psychologues, linguistes $\rangle\}$

c. $[[$ philosophes $\times E \cap$ Exlinguistes $]]=\{\langle$ philosophes, linguistes $\rangle\}$

Le domaine $E$, qui sert à établir une relation de paire, est naturellement apporté par les prédicats réciproques (ex. se respecter en $(2))^{13}$, les prédicats comitatifs (ex. coopérer en (19b)), et aussi les prédicats distributifs introduisant une dépendance épistémique. D'autre part, les prédicats distributifs qui prennent pour sujet des membres totalement indépendants (ex. être respectivement nés en (3b)) ne contribuent pas à fournir le domaine $E$, ce qui dégrade l'acceptabilité de la coordination nue même pour les conjoints stéréotypiquement reliés, comme mari et femme en (3b).

\footnotetext{
${ }^{13}$ Le Bruyn \& de Swart (2014) explique le fait que les conjoints nus, mari et femme en (2), sont interprétés, malgré l'absence de déterminant, comme le mari et sa femme, par Strongest Meaning Principle ('le principe du sens le plus fort'). Ce principe stipule que, lorsque le marquage explicite est absent, une interprétation plus forte, plus riche et plus typique est préférée à une autre moins forte, d'autant que cette interprétation est compatible avec le contexte. Ainsi le nom nu, femme, est interprété comme relié à mari, plutôt que comme une femme quelconque, car la première lecture est plus forte que la seconde lecture : celle-là implique celle-ci, mais l'inverse n'est pas vrai. Suivant la même logique, mari est interprété comme relié à femme, d'où la relation mutuelle entre les noms nus coordonnés.
} 


\section{Remarques récapitulatives}

Cette étude a montré que la coordination nue n'est pas toujours légitimée lorsque les conjoints construisent lexicalement une relation stéréotypique et conventionalisée, mais lorsque le prédicat verbal contribue à diviser les membres coordonnés et à établir une interdépendance entre eux.

Cette hypothèse permet de rendre compte des différences interprétatives entre la coordination nue et la coordination déterminée ainsi que de l'(in)compatibilité de la coordination nue avec différents prédicats pluriels : i) la coordination nue n'est pas compatible avec les véritables prédicats collectifs d'état comme être un couple en (47a), car ceux-ci exigent, pour leur sujet, une pluralité indivisible et ne permettent donc pas l'interdépendance des conjoints; ii) elle est compatible avec les prédicats inhéremment pluriels de type réciproque, comme se respecter en (47b), et de type comitatif, comme coopérer en $(47 \mathrm{c})$, car ces prédicats établissent, en permettant la permutation des arguments, une interdépendance des membres coordonnés ; iii) elle est compatible avec certains prédicats distributifs, comme diminuer le taux marginal supérieur d'impôt sur le revenu en (47d), car l'interdépendance est fournie par la dépendance épistémique établie entre les conjoints : les connaissances sur la politique fiscale de la France permettent au locuteur de prédire celle de l'Allemagne ; iv) elle est difficilement compatible avec d'autres prédicats distributifs, comme être respectivement nés en $(47 \mathrm{e})$, car les membres y sont totalement indépendants : on ne peut pas prédire du fait que le mari est né le 5 juillet 1944, que sa femme est née le 4 juin 1947.

(47)a. ??France et Allemagne sont un couple. (=(18)) [véritable prédicat collectif d'état]

b. Mari et femme se respectent toujours comme des invités honorés. $(=(2))$ [prédicat inhéremment pluriel de type réciproque]

c. France et Allemagne continuent de coopérer pour approfondir la construction européenne. $(=(19 b))$ [prédicat inhéremment pluriel de type comitatif]

d. Au cours des vingt dernières années, France et Allemagne ont diminué leur taux marginal supérieur d'impôt sur le revenu. (=(15a)) [prédicat distributif avec une dépendance épistémique]

e. ?Mari et femme sont respectivement nés le 5 juillet 1944 et le 4 juin 1947. $(=(3 b))$ [prédicat distributif sans dépendance épistémique]

Deux hypothèses ont ensuite été proposées pour comprendre pourquoi la coordination nue est assujettie à ces conditions légitimatrices : i) en français, les déterminants présupposent l'uniformité des membres. L'absence de déterminant indique inversement la non-uniformité des membres, ce qui implique nécessairement la division des membres. Cette hypothèse est appuyée par l'incompatibilité de la coordination nue avec une relative restrictive, qui sert à uniformiser les membres d'un groupe ; ii) en français, la coordination des noms nus est par défaut interprétée comme une intersection d'ensembles (ex. dans «Jean est linguiste et philosophe.»). Pour éviter à la lecture intersective, la coordination nue en position argumentale exige qu'une relation de paire soit établie entre les conjoints. C'est pour contribuer à établir une telle relation de paire que le prédicat verbal est contraint d'introduire une interdépendance des conjoints par la « symétrique logique » (définie par la permutabilité des arguments) ou par la «symétrie contextuelle » (réinterprétée en termes de la dépendance épistémique).

Par ailleurs, les travaux antérieurs ont montré que la coordination nue invoque, malgré l'absence de déterminant, diverses lectures, telles que le défini anaphorique, le défini unique, le générique, l'indéfini spécifique ou non spécifique, comme l'illustrent les 
exemples suivants.

(48) singulier / défini

a. Homme et voiture arrivent au camp. $(=(4 \mathrm{a}))$ [anaphorique / prédicat comitatif]

b. Quand on bascule la tête, une marine plus vraisemblable prend forme, où ciel et mer se remplacent réciproquement. (Lévi-Strauss, Tristes tropiques, 1955 : 79, cité par Curat, 1999 : 317) [unique / prédicat réciproque]

c. Mari et femme se respectent toujours comme des invités honorés. $(=(2))$ [générique / prédicat réciproque]

(49) pluriel / défini

a. Ils étaient à la limite de leurs forces. Soutiers et chauffeurs [co-rérérents à ils] [...] attaquaient au pic des monceaux de blocs. $(=(14 \mathrm{~b}))$ [anaphorique / prédicat implicitement comitatif]

b. Philosophes et linguistes se sont toujours accordés [...]. (=(1b)) [générique / prédicat réciproque]

(50) pluriel / indéfini [dans la plupart des cas, en objet]

a. Il composa vers et pastourelles à la manière ancienne. (Sollers, cité par Mulder, 2009) [spécifique / prédicat distributif]

b. Tous les linguistes consultent régulièrement grammaires pédagogiques et dictionnaires. (Roodenburg 2005 : 106) [non-spécifique / prédicat distributif]

Mulder (2009) montre que, dans son corpus incluant 43 cas attestés, 17 cas (39.54\%) incarnent la lecture définie anaphorique et 15 cas $(34.88 \%)$ donnent la lecture générique, tandis que 11 cas $(25.58 \%)$ transmettent la lecture indéfinie. Heycock \& Zamparelli (2003), Roodenburg (2004; 2005) et Le Bruyn \& de Swart (2014) font par ailleurs remarquer que la coordination nue des conjoints singuliers est le plus souvent interprétée comme définie, comme l'illustrent (48a-c).

Il conviendra de vérifier s'il y a une corrélation entre la lecture de la coordination nue et le type de prédicat verbal : ceci fera l'objet d'une étude future.

Je remercie Anne Zribi-Hertz de sa relecture attentive. Toutes les insuffisances qui restent sont dues à l'auteur. Cette étude a reçu le soutien financier de la Japan Society for the Promotion of Sciences (numéro 26370450).

\section{Références}

Benetti, L. (2008). L'article zéro en français contemporain : aspect syntaxique et sémantique. Bern : Peter Lang.

Brisson, C. (2003). Plural, all, and the nonuniformity of collective predication. Linguistics \& Philosophy, 26, 129-184.

Cadiot, P. (1997). Avec, ou le déploiement de l'éventail. In Guimier, C . (dir.). Cotexte et calcul du sens. Presses Universitaires de Caen, 135-155.

Curat, H. (1999). Les déterminants dans la référence nominale et conditions de leur absence. GenèveParis : Droz.

Gary-Prieur, M.-N. (2011). Les déterminants du français. Paris : Orhrys.

Hackl, M. (2002). The Ingredients of Essentially Plural Predicates. In Hirotani, M. (ed.). Proceedings of NELS, 32, 171-182.

Haspelmath, M. (2007). Coordination. In Shopen, T. (ed.). Language Typology and Syntactic Description. Cambridge : Cambridge University Press, 1-51.

Heycock, C. \& Zamparelli, R. (2003). Coordinated Bare Definites. Linguistic Inquiry, 34, 443-469. 
Jayez, J. \& Mari, A. (2005). Togetherness. In Maier E., Bary, C. \& Huitink, J. (eds). Proceedings of Sinn und Bedeutung, 9, 155-169.

Lambrecht, K. (1984). Formulaicity, Frame Semantics and Pragmatics in German Binominal Expressions. Language, 60.4, 753-796.

Lasersohn, P. (1998). Events in the Semantics of Collectivizing Adverbials. In Rothstein, S. (ed.) Events and Grammar. Dordrecht : Kluwer, 273-292.

Le Bruyn, B. \& de Swart, H. (2014). Bare coordination: the semantic shift. Natural Languages \& Linguistic Theory, 32, 1205-1246.

Mari, A. (2005a). Sous-spécification et interprétation contextuelle : le cas de avec. In Corblin, F. \& Gardent, C. (éds.). Interpréter en Contexte. Lavoisier, 81-106.

Mari, A. (2005b). Intensional epistemic wholes : A study in the ontology of collectivity. In Machery, E. \& Werning, M. (eds.). The compositionality of Meaning and Context, Vol. 1 Foundational Issues. Ontos Verlag, 189-212.

Mari, A. (2012). A note on generic quantification and the ontology of twins and bikini. In Mari, A. (dir). Recherches linguistiques de Vincennes, 41, 147-162.

Märzhäuser, C. (2013). Coordinated bare nouns in French, Spanish and European Portuguese. In Kabatek, J. \& Wall, A. (eds.). New Perspectives on Bare Noun Phrases in Romance and Beyond. Amsterdam : John Benjamins, 283-300.

Märzhäuser, C. (2014). L'interfaces de la syntaxe dans l'analyse de la construction des noms nus coordonnés. SHS Web of Conferences 8, 4ème Congrès Mondial de Linguistique Française, 25012513, http://dx.doi.org/10.1051/shsconf/20140801387

McNally, L. (1993). Comitative coordination: a case study in group formation. Natural Language \& Linguistic Theory, 11, 347-379.

Michaux, C. (1998). Reducing the Coordination of Determiners: Some Principle. In Hamm, F. \& Hinrichs, E. (eds.). Plurality and Quantification. Dordrecht : Kluwer, 321-337.

Milner, J.-C. (1979). De la syntaxe à l'interprétation. Paris: Seuil, 1979.

Moltmann, F. (2004). The Semantics of Together. Natural Language Semantics, 12, 289-318.

Mulder, V. (2009). Les noms nus coordonnés: Les $N$ et $N$ du français. Mémoire de licence, l'Université de Utrecht. http://www.hum.uu.nl/medewerkers/b.s.w.lebruyn/weakreferentiality/files/Mulder_bachelor.pdf

Riegel, M., Pellat, J.-C. \& Rioul, M. (1999). Grammaire méthodique du français. Paris : PUF.

Roodenburg, J. (2004). French Bare Arguments Are Not Extinct: The Case of Coordinated Bare Nouns. Linguistic Inquiry, 35, 301-313.

Roodenburg, J. (2005). Une coordination particulière : les syntagmes $N$ Conj $N$ en français. Langages, 160, 93-109. 